\title{
OESOPHAGITIS IN INFANCY
}

BY

\author{
J. H. EBBS, M.D., D.C.H.
}

(From the Children's Hospital, Birmingham)

The majority of text-books dealing with diseases of children, written during the past fifty years, describe oesophagitis as an inflammation which occurs : $(a)$ as the result of mechanical or chemical irritation, $(b)$ in the catarrhal stage of the specific fevers, $(c)$ rarely as an extension of an infection in the mouth and (d) occasionally spontaneously in young infants. During infancy oesophagitis does not seem to have attracted much attention. Its presence in a number of post-mortem examinations in infants and the recognition of this disease in a number of infants during hospitalization have provided sufficient material to make a brief survey of the condition. A review of the records of the Birmingham Children's Hospital for the past three years shows that whereas thirty-one children have been admitted for treatment of foreign bodies in the oesophagus, poisoning and congenital strictures, twenty-eight children have died with an acute oesophagitis. The age of the former group was between one and twelve years. Those cases of acute oesophagitis in the latter group were under ten months of age, the average being four months.

Twenty-eight cases of acute oesophagitis are classified in the following table according to their etiology.

\section{Table 1}

\begin{tabular}{|c|c|c|c|c|c|}
\hline THRUSH & $\cdots$ & $\cdots$ & . & $\cdots$ & 22 \\
\hline NON-SPECIFIC & . & . & . & $\cdots$ & 3 \\
\hline DIPHTHERIA & . & . & . & . & \\
\hline TUBERCULOSIS & . & . & . & . & \\
\hline VINCENT'S ORC & ISM & $\ldots$ & $\ldots$ & $\ldots$ & \\
\hline
\end{tabular}

Those cases recorded as non-specific were found to have ulceration and inflammation of the oesophagus without a specific organism or etiological agent being demonstrated.

Table 2 has been compiled to record the cases with the age, chief complaint, important clinical points and principal pathological findings.

\section{Clinical features}

Although the complaint of pain beneath the sternum or on swallowing cannot be made by infants, nevertheless the outstanding observation of the mother or nurse in acute oesophagitis in infants is that the infant cries during 


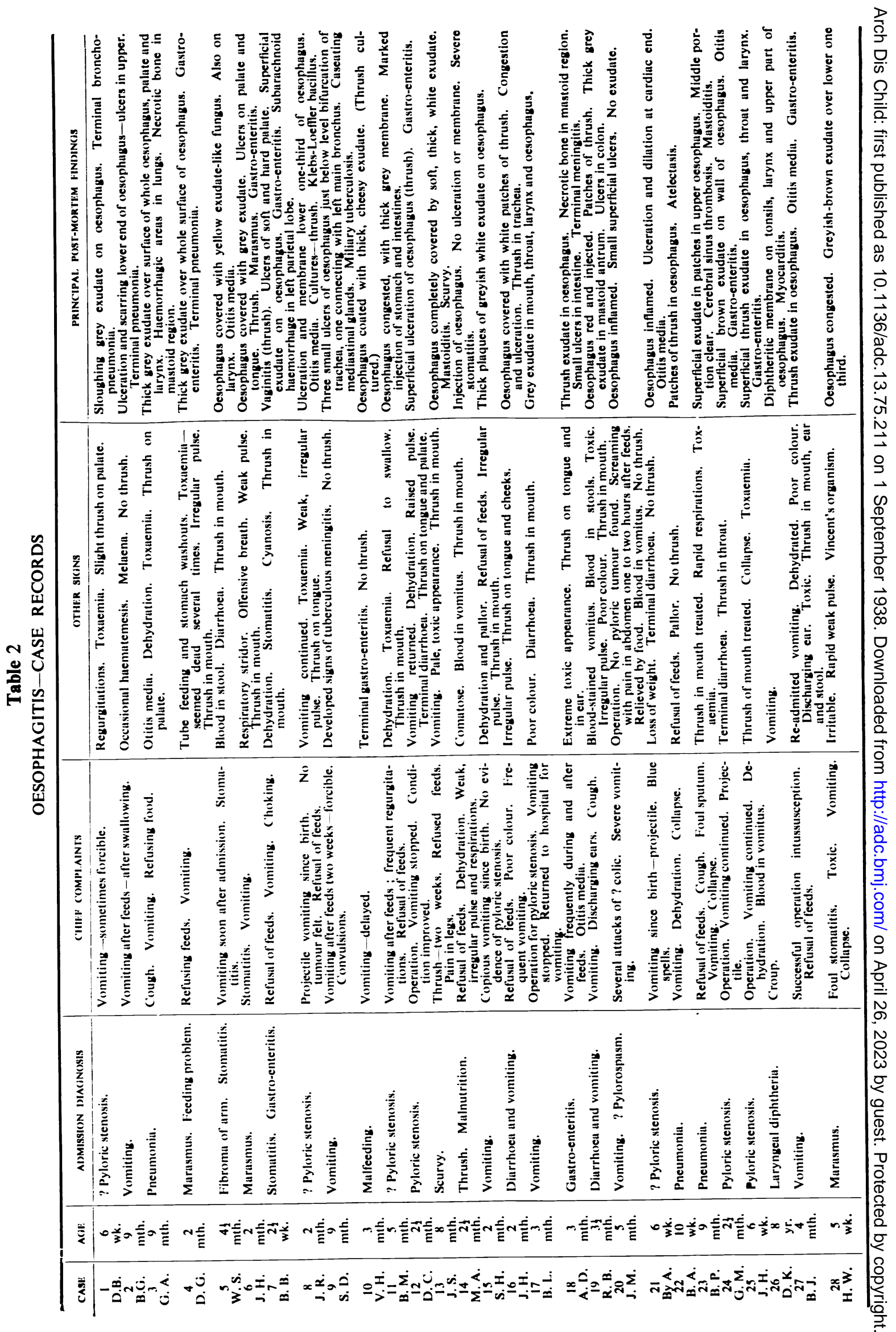


the act of swallowing or immediately after it swallows, or that it refuses to feed. Many of the infants in this series would take the liquid into the mouth and then allow it to dribble from the corners. The chief complaint was vomiting, this

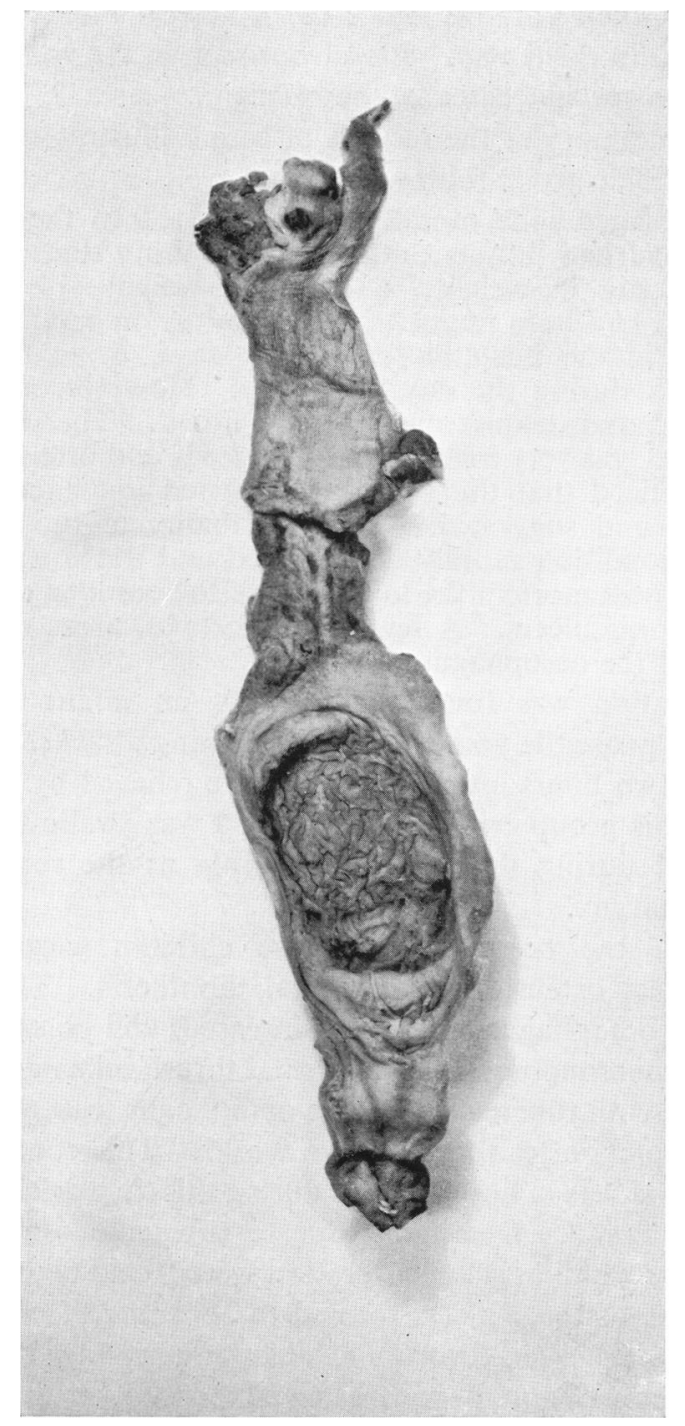

FIG. 1.-Case 8. Oesophagus and stomach. Ulceration and membrane. Diphtheria and thrush.

being of a particular type in that the food if swallowed was vomited back either as an immediate regurgitation of the food taken or as a large, forceful vomit during, immediately after or within a short time after the feed. The forceful vomiting has resulted in several infants being suspected of having pyloric 
stenosis. Eleven infants were investigated with this condition in mind. In four cases of pyloric stenosis, thrush oesophagitis followed the operation. Projectile or forcible large vomits were noted in six other infants in this group.

Blood was noticed in the vomitus of nine infants and melaena was present in four. Dark brown vomitus occurred in several others and suggests that blood was present in them also. Blood was seen in the vomitus of all three of the so-called non-specific cases of oesophagitis (cases 2, 20 and 21). The following short summary of the records of case 2 illustrates the type of course which the non-specific group followed.

Case 2, B.G., aged nine months, was admitted to the hospital with the history that he had had frequent attacks of vomiting since birth. There had been very little gain in weight. (Admission weight-14 lb. $6 \mathrm{oz}$.) Small amounts of blood had been noticed in the vomitus on several occasions. The infant improved in the hospital for several days, being fed small frequent quantities of milk during the day and night. However, after one week the vomiting returned and definite melaena was noted. The vomitus was bloodtinged. The child was very irritable between feeds and often cried as though in pain. It was noticed that the vomiting occurred immediately after the first swallow of food and suggested some abnormality of the oesophagus. The infant's general condition rapidly deteriorated and death was due to terminal pneumonia. At post mortem the lower end of the oesophagus was scarred and covered with chronic ulcers. A few small ulcerated areas were present in the middle portion of the oesophagus.

Similar ulceration was found in case 20 -an infant of four-and-a-half months who had projectile vomiting and screaming attacks. The latter would come on one to two hours after feeds and were relieved by taking food. The other infant in this group cried when the food was swallowed and sometimes became cyanosed during the feed, presumably as the result of milk being retained in the pharynx.

Diphtheria of the oesophagus in two children (cases 8 and 26) was the result of direct extension of laryngeal diphtheria. The membrane and ulceration of the oesophagus and larynx in case 8 will be seen in fig. 1. This child also had a superimposed or coincidental thrush infection.

The one child with tuberculosis of the oesophagus (case 9) gave a history of vomiting immediately after food for two weeks. This continued for the two weeks that the child was in hospital, and during this time evidence of generalized tuberculosis was found. Death was due to tuberculous meningitis. At the post mortem three small areas of ulceration were found near the middle of the oesophagus. One of these ulcers formed the opening of a fistula which connected with the left main bronchus. Large caseating tuberculous glands were found in this region and appeared to be the cause of the oesophagitis. A section of the wall of the oesophagus at the site of one of the ulcers is shown in fig. 2.

The oesophagus of the child with Vincent's infection was covered with a brown, stringy exudate which had formed on top of superficial ulcers scattered on the whole surface. These ulcers had clearly marked edges with a surrounding ring of inflammation.

Thrush was the parasitic organism found in the direct smears and cultures 


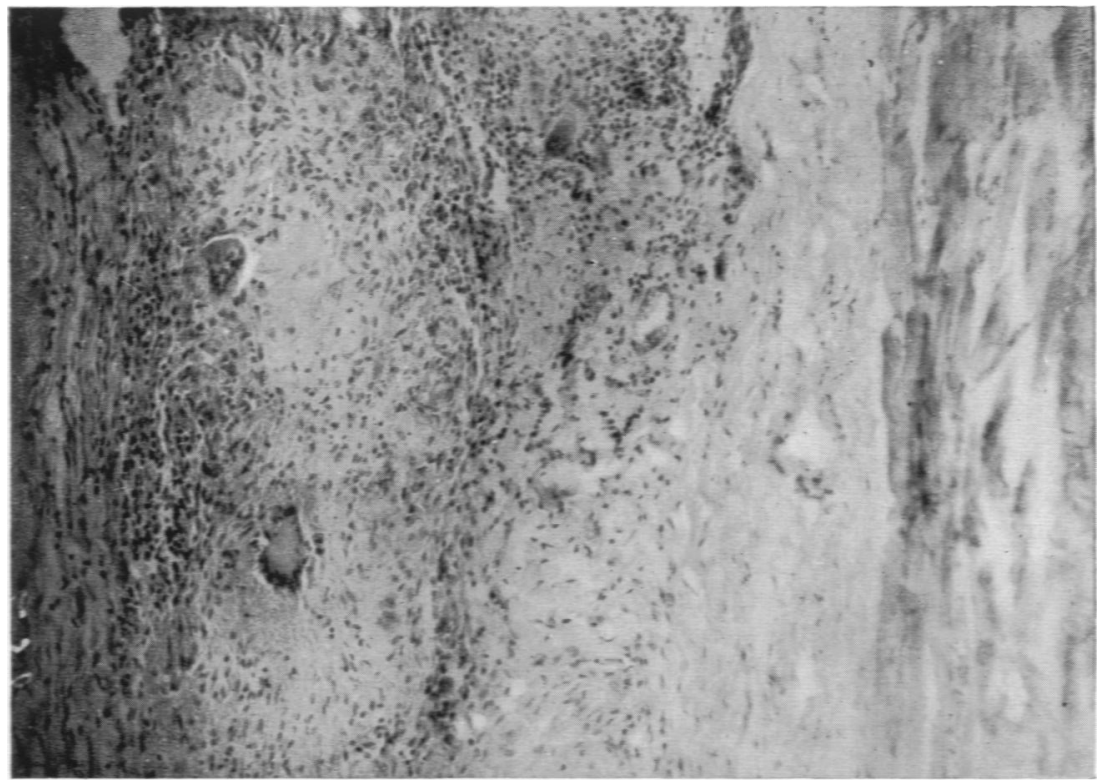

Fig. 2.-Case 9. Tuberculosis of the oesophagus. Section of wall of oesophagus through area of ulceration. $\times 120$. H. \& E.

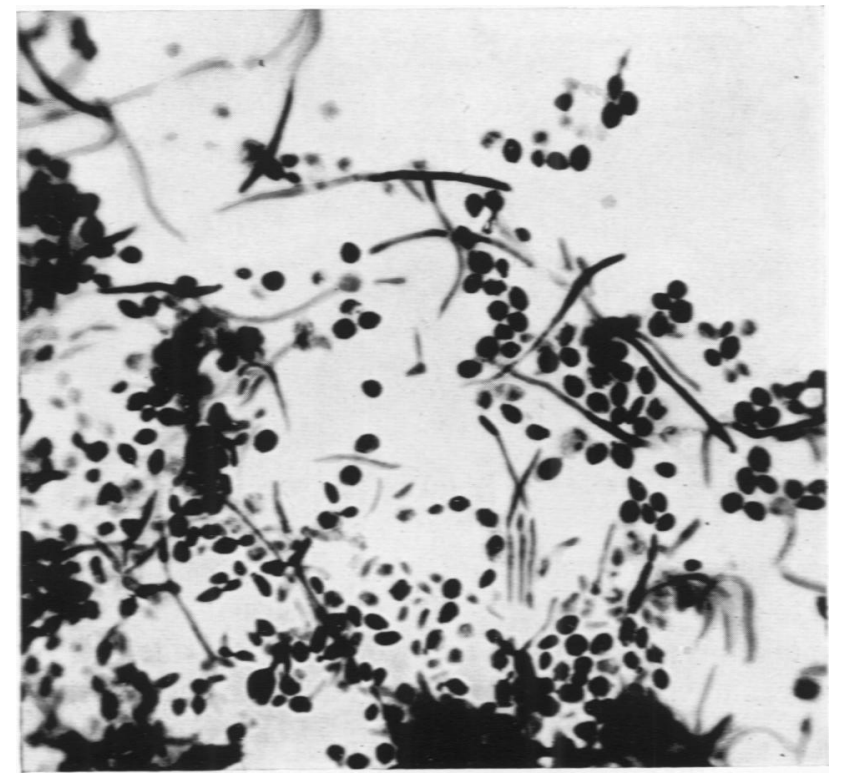

FIG. 3.-Case 27. Section of gelatin culture of thrush. Gram stain ; $\times 660$. 
of twenty-two infants with oesophagitis. They had, with a few exceptions, been noted to have oral thrush. Apart from the common symptom of vomiting, these infants with thrush infection presented a clinical picture which seemed to be characteristic in many respects, and enabled a diagnosis of thrush oesophagitis to be made in several cases. A previous history of thrush of the mouth or its presence during examination was usually noted. Refusal to take feeds was a very common complaint, and was presumably due to the sore tongue and

Fig. 4.-Case 12. Oesophagus with patches of thrush and early ulceration.

mouth caused by thrush. In a few infants there was a refusal to swallow, since this appeared to cause marked pain. They were usually irritable. Milk would sometimes be taken into the mouth and would then be allowed to run out with no attempt to swallow. If food was swallowed it was noted in most cases that this was regurgitated immediately. In other cases the milk would be vomited back in a projectile manner or forcibly soon after feeding. Streaks of blood were frequently observed in the vomitus. Dehydration became a serious factor 
in the presence of frequent vomiting, and parenteral fluid administration was usually required. The loss in weight sometimes became alarming.

Toxaemia appeared to develop, usually a few days after the onset of severe symptoms. In spite of seemingly adequate parenteral fluid and correction of

Fig. 5.-Case 13. Membrane, ulceration and haemorrhage in thrush oesophagitis.

the dehydration, and without signs of infection elsewhere in the body, these infants would become a pale greyish colour, with cold and sometimes cyanotic extremities. The temperature in some cases was slightly raised, but was often subnormal. The pulse rate was always rapid and usually irregular. The 
volume in some cases was so weak that records of pulse could not be made. Respirations were irregular, but usually only became rapid in the terminal stages. Periods of apparent recovery with less vomiting, brighter appearance and colour, alternated with periods when the child appeared to be dying. Diarrhoea was never an outstanding sign. Extension of the thrush infection to the intestines

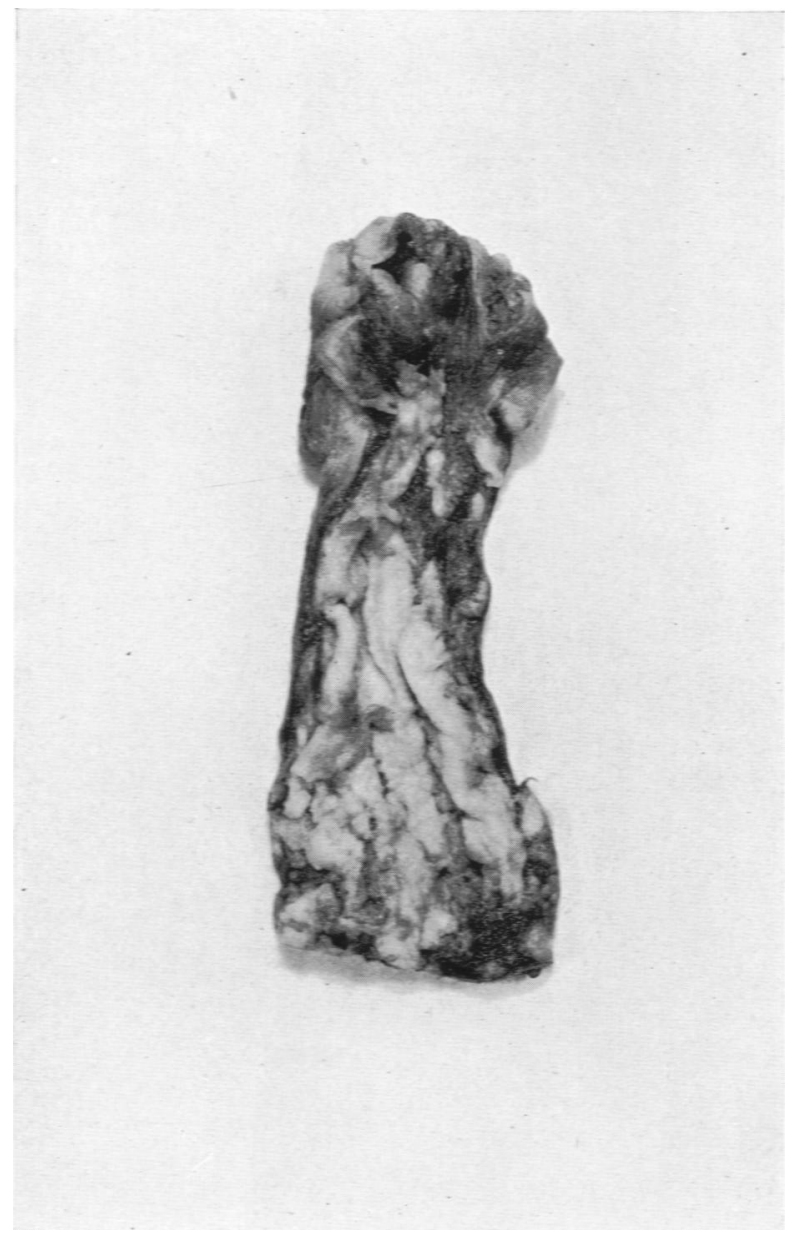

FIG. 6.-Case 4. Thick white exudate with ulceration and haemorrhage in severe thrush oesophagitis.

was demonstrated in two cases. Terminal broncho-pneumonia occurred in nearly every case.

The predisposing factors in the occurrence of this form of infection are theoretical but certain common facts were noted. All of the infants were artificially fed. Several cases were associated with debilitating conditions, such as pyloric stenosis, otitis media and previous attacks of gastro-enteritis. Stomach wash-outs were employed in eight infants, including the cases of 


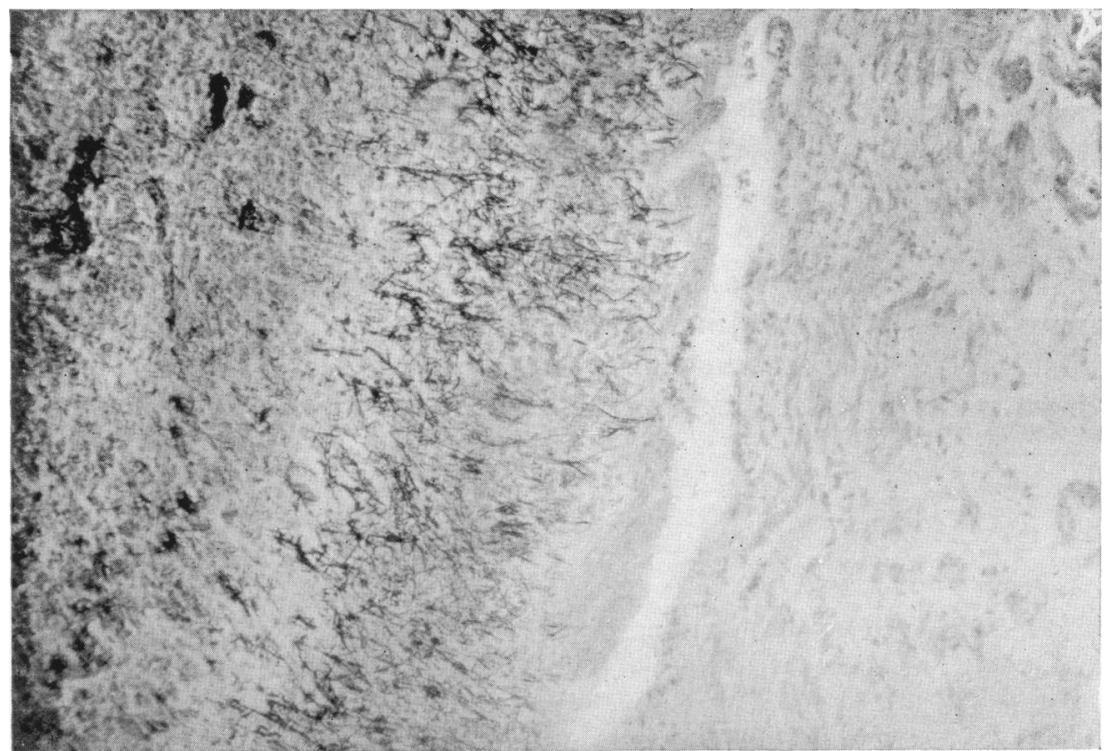

Fig. 7.-Section of wall of oesophagus. Thrush mycelium penetrating the tissues. Gram stain ; $\times 120$.

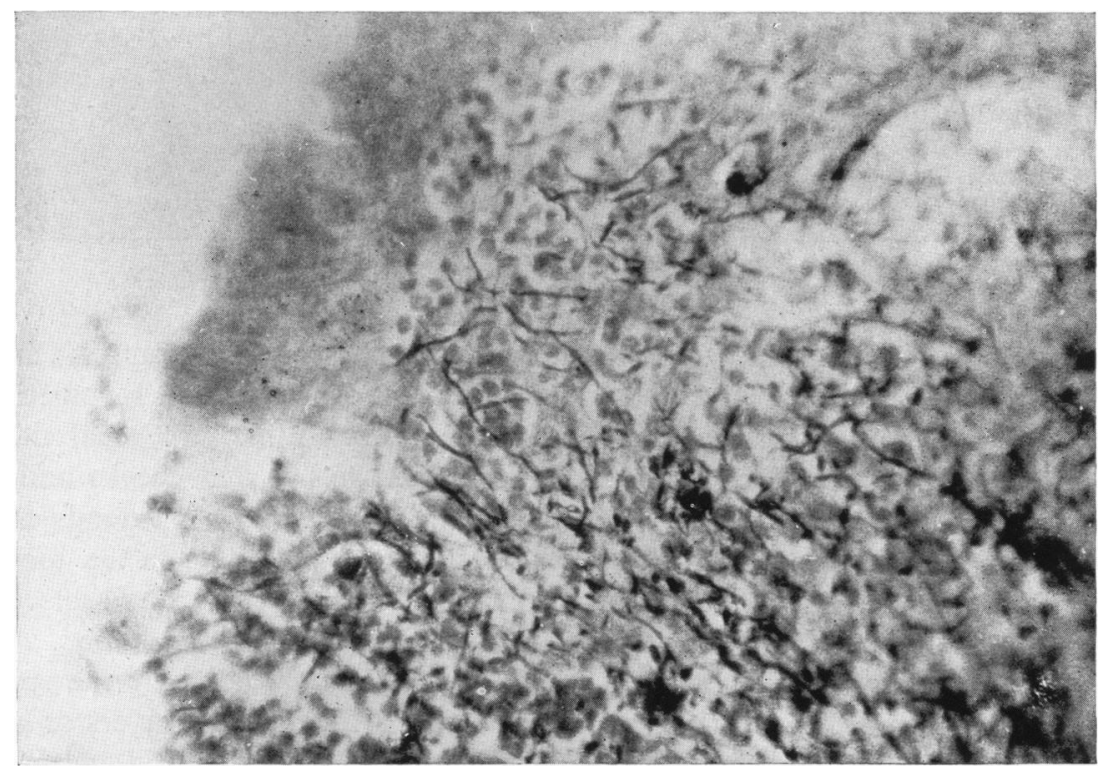

FIG. 8.-Thrush oesophagitis. Ulceration of surface epithelium and growth of mycelium in deeper layers. Gram stain.; $\times 290$. 
pyloric stenosis, and the possibility of trauma to the oesophagus, which would allow the thrush infection to become established, cannot be ignored. The passage of any instrument through the mouth of a young infant with thrush appears to be attended by a definite risk of spreading the infection into the oesophagus. This possibility is a serious one in cases of pyloric stenosis.

\section{Pathology}

Thrush is a parasitic disease caused by a fungus which has been given a variety of names, but which is commonly called monilia albicans. So-called ' white mouth' is a not uncommon finding in young artificially-fed infants living in poor hygienic surroundings, and is found with many protracted wasting

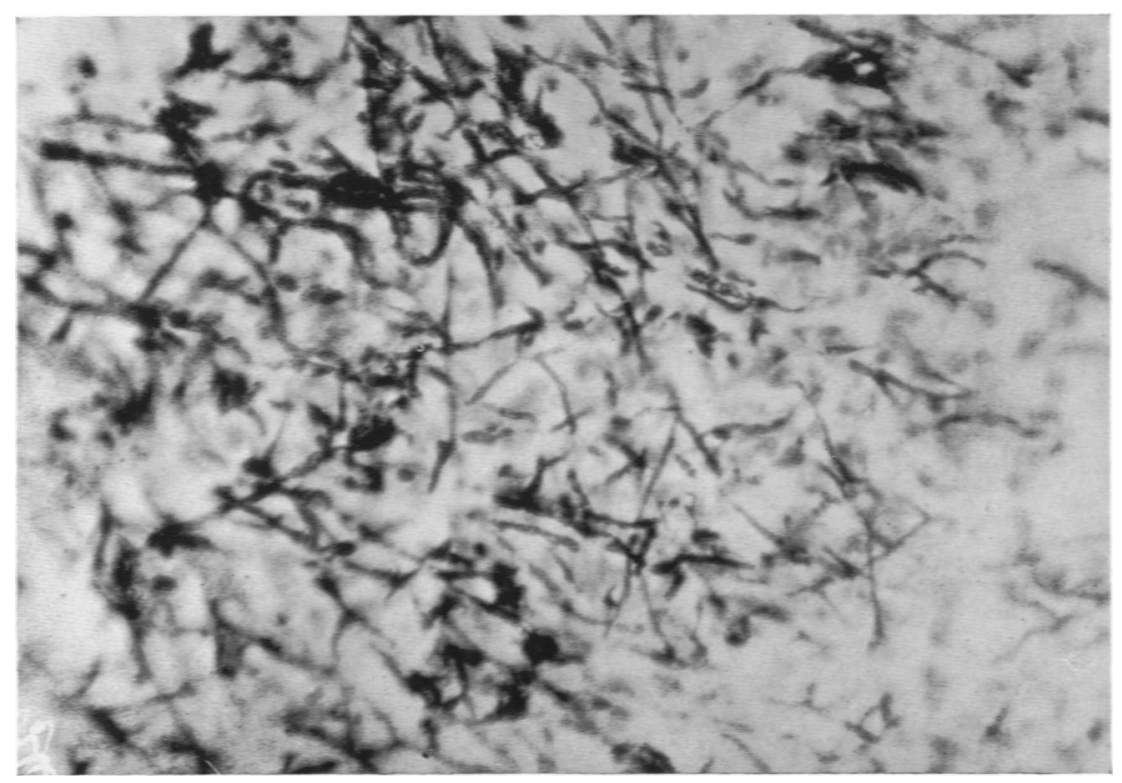

FIG. 9.-High-power of fig. 8.

diseases of early life. The disease seldom develops on healthy mucous membrane, its growth being favoured by slight abrasions or irritation. It sometimes occurs in mild epidemics in institutional nurseries. While the usual site of thrush is the margin of the tongue, the inside of the lips and cheeks and the palate, it not infrequently involves the whole pharynx and rarely extends down the oesophagus or into the larynx. Small white patches appear in the mouth, which look like clotted milk but which when rubbed off leave small bleeding points. The mouth and tongue is usually dry and slightly inflamed. The infection is a superficial ulceration which is apparently caused by the spores lodging on the surface and then spreading between the different layers of cells. These spores are oval or oblong in shape, about $5 \mu$ to $7 \mu$ in size, and are readily seen in direct smears from the lesions stained by ordinary methods. The mycelial form of the fungus is a large, bacillary, septate organism $20 \mu$ to $30 \mu$ 
long and $4 \mu$ to $5 \mu$ in diameter. These organisms grow on the majority of ordinary culture media. A section of a gelatin culture is photographed in fig. 3 .

In the oesophagus the lesion is a superficial ulceration with a soft, thick, grey exudate over the surface which can be partially removed fairly easily (fig. 4). The longer the infection has been present the more adherent the exudate becomes, and in a few cases could not be removed without stripping the whole epithelial surface (figs. 5 and 6).

Histological sections of the wall of the oesophagus at the site of the infection show a superficial ulceration with an exudate or membrane composed of spores, epithelial cells and debris. The mycelium can be seen penetrating the wall,

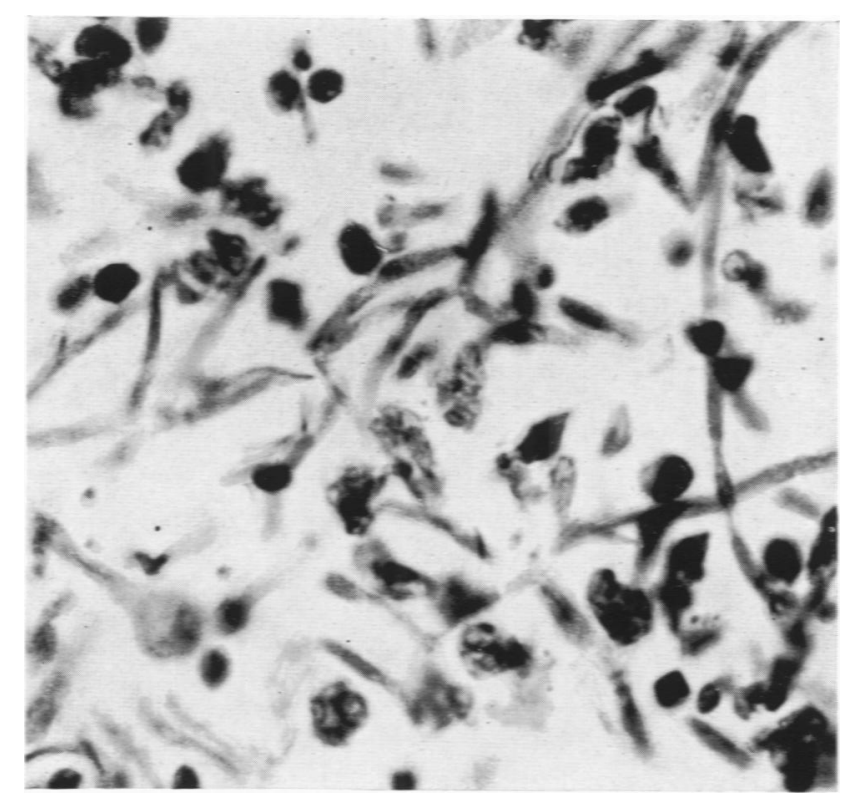

FIG. 10.-Section of wall of oesophagus showing mycelium and spore formation with several degenerating epithelial cells. Gram stain ; $\times 1100$.

lying between the epithelial cells, entering the submucous layer, the muscularis mucosa and in a few cases the muscle coat. The capillaries in the submucosa show marked congestion. (Photographs illustrating the histological picture are shown in figs. 7, 8, 9 and 10.)

Patients with superficial lesions have been described who have had agglutinins in their blood (Gay and others, 1935). Holt and McIntosh (1933) state that thrush may invade the blood and cause thrombosis or be carried to distant parts of the body. Monilia infection has been described in many parts of the body (Eustace Smith, 1884). In the group of patients observed in this series with oral thrush and oesophagitis, there were two infants with small pinpoint ulcers in the colon in whose stools large numbers of thrush spores were found. One female infant also had a severe membranous vaginitis which 
was due to the same organism, another child had a balanitis, one a conjunctivitis and two with pneumonia had thrush in the lungs. Two with otitis media

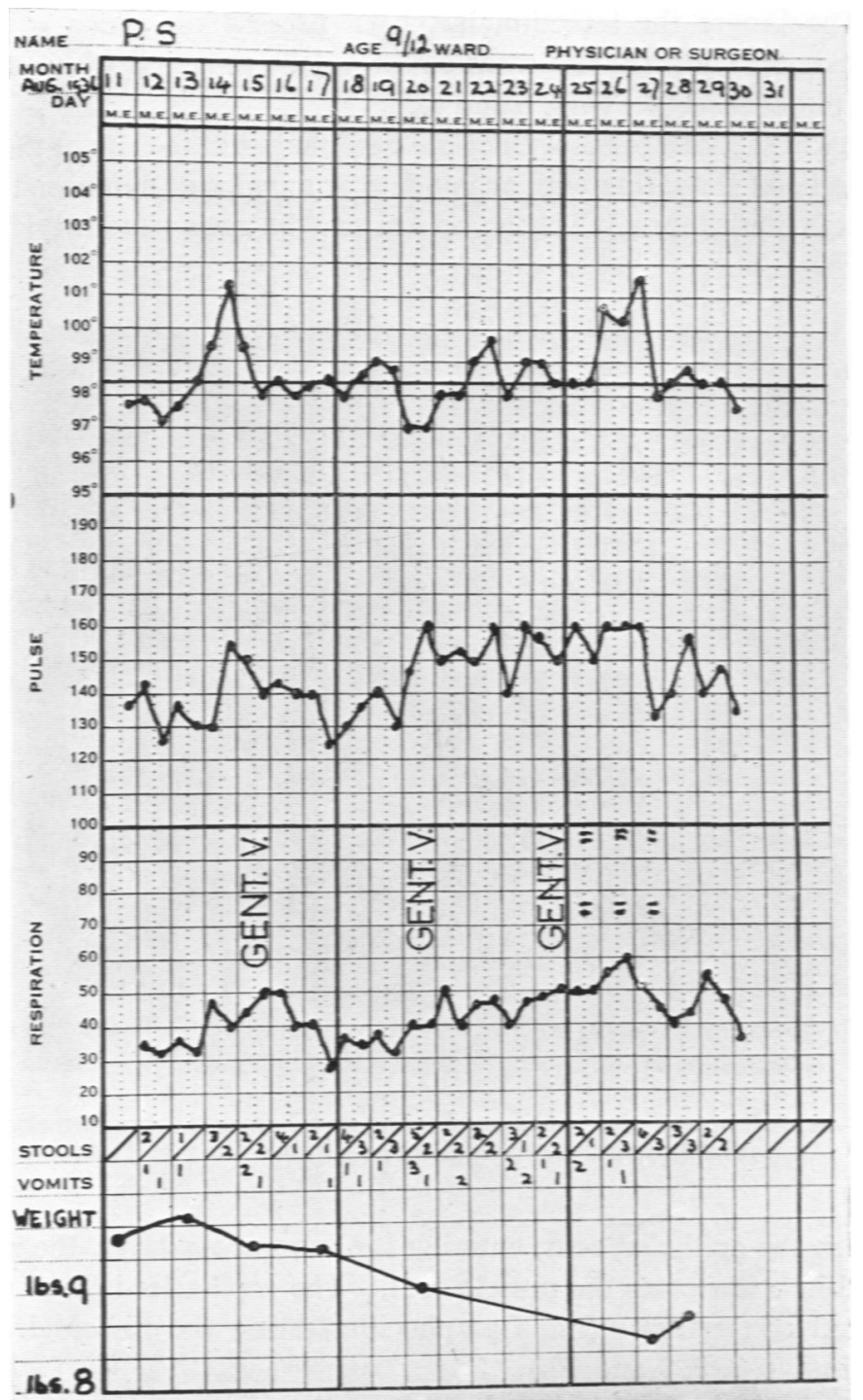

FIG. 11.-Temperature chart of Case P.S. showing clinical course with gentian violet traatment. Gent. V.= mouth painted with gentian violet (1 per cent. aqueous solution).

had large numbers of thrush spores in the discharge, and at post mortem there was a mass of grey purulent exudate and the bone in the mastoid region was necrotic. 


\section{Treatment}

The treatment of thrush consists in local applications, oral hygiene and attention to the general health of the infant. A 1 per cent. aqueous solution of gentian violet has been found satisfactory in hospital cases. Borax and glycerin has been disappointing. Signs of the disease in the mouth usually disappear after the first application of gentian violet. The only disadvantage appears to be the temporary staining of the lips and mouth. Liberal applications of gentian violet have been applied to the mouth in suspected cases of thrush oesophagitis in the hope that it would reach the affected areas in the oesophagus. The results have been encouraging, as illustrated by the following case record.

P.S., aged nine months, was brought to the hospital for the treatment of a naevus, and on routine examination was found to have thrush, which disappeared after one application of gentian violet. Two days later the temperature became elevated and vomiting commenced for which no adequate cause could be found. Another application of gentian violet to the mouth resulted in the vomiting stopping for two days. It returned again, and since there was no further sign of thrush in the mouth it was thought to be due to some other cause, although none was demonstrated. The child's condition became extremely critical, the skin was grey, frequent vomiting and slight diarrhoea produced dehydration and in spite of adequate fluid therapy the child appeared several times as if she were about to die. Experience with other patients in whom this peculiar toxic appearance associated with vomiting and following a previous history of thrush had been noted, made the diagnosis of oesophagitis likely. Following several liberal applications of gentian violet to the mouth the vomiting became less, the general condition improved and the child was finally discharged in good condition and gaining weight. (See temperature chart, fig. 11.)

An infant who refuses feeds and vomits soon after swallowing, who has had a thrush infection of the mouth, and who shows a deterioration of its general condition, with a toxaemia which is out of all proportion to the signs which are present, should be suspected of thrush oesophagitis and should be treated as such.

\section{Summary}

Twenty-eight cases of oesophagitis in infancy have been briefly reviewed. Twenty-two of these were caused by thrush and presented a clinical syndrome in which the outstanding signs and symptoms were the presence of oral thrush, refusal to take feeds, vomiting during or soon after feeding, severe toxaemia, with pallor and weak pulse and in some cases the presence of blood in the vomitus, and in a few melaena. The pathological process was one of ulceration of the epithelium and invasion of the submucosa by the mycelium of the thrush fungus. Two cases of diphtheria of the oesophagus, one of tuberculosis and one due to Vincent's organism were found. Three infants have been classified as non-specific who were found to have ulcers at the lower end of the oesophagus and who had pain during or after feeding. Local treatment of oral thrush with 
gentian violet appears to be satisfactory and one case of oesophagitis is described which appeared to respond to this form of treatment. Thrush is not the benign condition which it is commonly thought to be.

\section{REFERENCES}

Gay, F. P., and associates (1935). Agents of Disease and Host Resistance, London, 1,120. Holt, E. L., Jr., and McIntosh, R. (1933). Holt's Diseases of Infancy and Childhood, London, 356.

Smith, E. (1884). Disease in Children, London, 572. 